\title{
DYNAMICS OF A DRIVER BEAM PROPAGATING IN AN UNDERDENSE PLASMA WITH A DOWNWARD DENSITY TRANSITION
}

\author{
H. Suk ${ }^{*}$, N. Barov, J. England, E. Esarey ${ }^{\dagger}$, G. Kim ${ }^{*}$, J. Rosenzweig, and M. Thompson \\ KERI, Changwon, Kyungnam 641-120, Rep. of Korea \\ Department of Physics and Astronomy, UCLA, Los Angeles, CA 90095, USA \\ ${ }^{\dagger}$ Center for Beam Physics, LBNL, Berkeley, CA 94720, USA
}

\begin{abstract}
When a short electron beam propagates in an underdense plasma (plasma density $n_{0}<$ beam density $n_{b}$ ) with a downward density transition, it is known that some background plasma electrons are trapped and accelerated by the plasma wakefield [1]. Beam quality of the trapped plasma electrons is severely affected by the wakefield that is generated by the driving electron beam, so dynamics and instabilities of the driver beam are very important. In this paper, we present some simulation results on the self-trapping and driver beam dynamics.
\end{abstract}

\section{INTRODUCTION}

Plasma-based advanced accelerators [2-5] have been studied extensively as they can provide a much higher acceleration gradient than conventional RF-based accelerators. However, beam injection for acceleration is a difficult process so that it has been one of critical issues in this field. In order to avoid such a problem, Bulanov et $a l$. [6] proposed to use a gently decreasing plasma density profile for self-trapping of plasma electrons, in which the density scale length $L_{s}$ is much greater than the plasma wavelength $\lambda_{p}$. In this scheme, some plasma electrons from wave breaking are trapped and accelerated by the wakefield. On the other hand, Suk et al. [1] proposed the use of a sharply-downward density transition for selftrapping, in which $L_{s}$ is much less than $\lambda_{p}$. In this case, some background plasma electrons are self-injected into the acceleration phase of the wakefield when the wake wave passes the density transition. The trapped plasma electrons, named plasma electron beam, are then accelerated to a high energy by the wakefield and a fairly good beam quality is expected [7]. In case that the wake wave is generated by a high-energy electron beam, the wakefield is gradually deformed as the driver beam loses its energy to the plasma. Thus quality of the plasma electron beam is severely affected by dynamics of the driver beam. In this paper, we present 2-dimensional simulation results on the self-trapping and the driver beam dynamics.

*Email address: hysuk@keri.re.kr

\section{TRAPPING OF BACKGROUND PLASMA ELECTRONS}

In order to study the trapping phenomenon, we used the 2-dimensional MAGIC code [8] that is a fully relativistic and electromagnetic particle-in-cell code. In the simulation, the driver beam has a bi-Gaussian density profile $n_{b}(r, z)=n_{b, 0} e^{-r^{2} / 2 \sigma_{r}^{2}} e^{-\xi^{2} / 2 \sigma_{z}^{2}}\left(\xi=z-v_{b} t\right)$ and it propagates through an underdense plasma with a downward density transition $\left(n_{b, 0}=2.9 n_{0}{ }^{I I}=2 n_{0}{ }^{I}\right)$. When an electron beam enters a sharp vacuum-plasma boundary, some plasma electrons are trapped by the wakefield. But these electrons have a large energy spread and the charge is small. In order to avoid such an unnecessary trapping at the vacuum-plasma boundary, a slowly increasing density profile, as shown in Fig. 1(a), is used in the simulation, in which the density gradient scale is larger than $\lambda_{p}$. Therefore, when the electron beam enters the vacuum-plasma boundary, plasma electrons are adiabatically expelled so that trapping of the stray electrons at the boundary can be avoided. Since the driver beam is short $\left(k_{p} \sigma_{z}{ }^{I I}<2\right)$, it excites a highly nonlinear and strong wakefield. When the wake wave passes the density transition, a significant amount of background plasma electrons are trapped by the wakefield as the plasma wavelength becomes larger at the downward density transition.

Table 1: Beam and plasma parameters for the simulation.

\begin{tabular}{|l|c|}
\hline Beam energy $E_{b}$ & $16 \mathrm{MeV}$ \\
\hline Peak beam density $n_{b, 0}$ & $10^{14} \mathrm{~cm}^{-3}$ \\
\hline Beam radius $\sigma_{r}$ & $450 \mu \mathrm{m}\left(k_{p} \sigma_{r}{ }^{I I}=0.5\right)$ \\
\hline Beam length $\sigma_{z}$ & $1 \mathrm{~mm}\left(k_{p} \sigma_{z}{ }^{I I}=1.1\right)$ \\
\hline Plasma density $n_{0}$ & $n_{0}{ }^{I}=5 \times 10^{13} \mathrm{~cm}^{-3}$ \\
$n_{0}{ }^{I I}=3.5 \times 10^{13} \mathrm{~cm}^{-3}$ \\
\hline $\begin{array}{c}\text { Plasma electron } \\
\text { temperature } k T_{e}\end{array}$ & $3 \mathrm{eV}$ \\
\hline
\end{tabular}


(a)

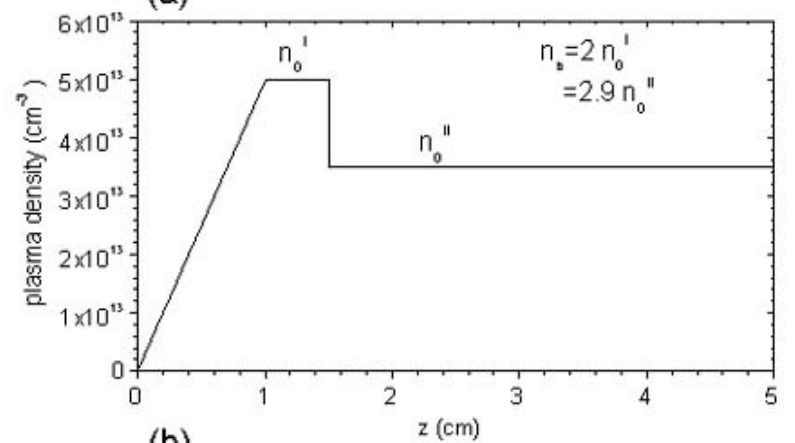

(b)

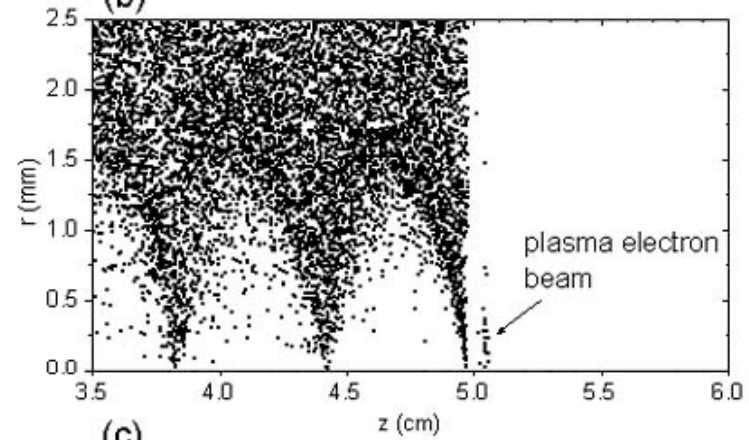

(c)

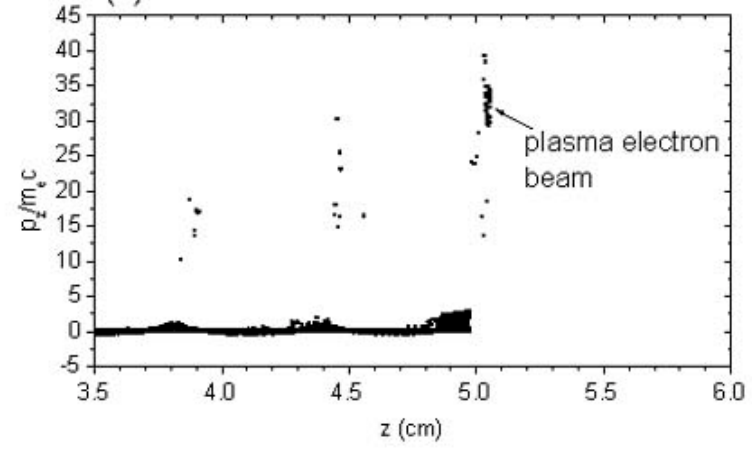

Figure 1: (a) Plasma density profile for the simulation. The plasma particles are distributed up to $z=5 \mathrm{~cm}$. (b) Plot of phases space $(r, z)$ for the plasma electrons. (c) Plot of phase space $\left(p_{z}, z\right)$ for the plasma electrons.

The trapped plasma electron beam is accelerated in a plasma-electron-free ion channel that provides a linear focusing force to the beam, which preserves an emittance. This is one of the reasons why a good beam quality can be achieved with the present trapping scheme. As the driver beam propagates, the wake wave follows it. When the driver beam exits from the plasma, the wake wave disappears beyond the plasma boundary. However, the trapped plasma electron beam has a high energy already so that it continues to propagate. This situation is shown in Fig. 1(b), in which the first plasma oscillation period passed the plasma boundary and it disappeared already, while the plasma electron beam is moving out of the background plasma. This phenomenon is shown in Fig. 1 (c) more clearly. The figure indicates that the plasma electron beam has an energy of about $16 \mathrm{MeV}$ (this is the same as the driver beam energy), while the background plasma electrons are just oscillatory.

\section{DYNAMICS OF THE DRIVER BEAM}

When an electron beam propagates in a plasma, it is well known that a number of instabilities can occur in the beam. For example, such instabilities as the transverse two-stream instability, electron-hose instability, etc., can be developed if the beam length $L_{b}$ is larger than $\lambda_{p}$ [9]. Generally a long beam tends to be more detrimental to instabilities. That is why a short-pulse driver is preferred for wakefield accelerations. However, the simulation shows that even a short electron beam with $L_{b}<\lambda_{p}$ can develop an instability as the beam loses its energy. The result is shown in Fig. 2. For this simulation, the same beam and plasma parameters as in Sect. 2 were used. But this time the plasma distribution extends beyond $z=5 \mathrm{~cm}$ so that the driver beam in Fig. 2 is still in the plasma region. Figure 2 shows that the driver beam loses its energy as it propagates through the plasma and it deforms longitudinally. In addition, the figure indicates that the driver beam develops a high frequency instability of which the frequency is much higher than the plasma oscillation frequency $\omega_{p}$ and the betatron frequency $\omega_{\beta}$. Here, the betatron frequency is given by $\omega_{\beta}=\omega_{p} / \sqrt{2} \gamma$ and the parameter $\gamma$ is the usual Lorentz factor. It seems that the high frequency instability is associated with the betatron radiation. As is well known, the head part of the beam is still immersed in the plasma, but the rest part of the beam is in an ion channel. The beam electrons in the ion channel region execute transverse betatron oscillations and this motion leads to the betatron radiation.

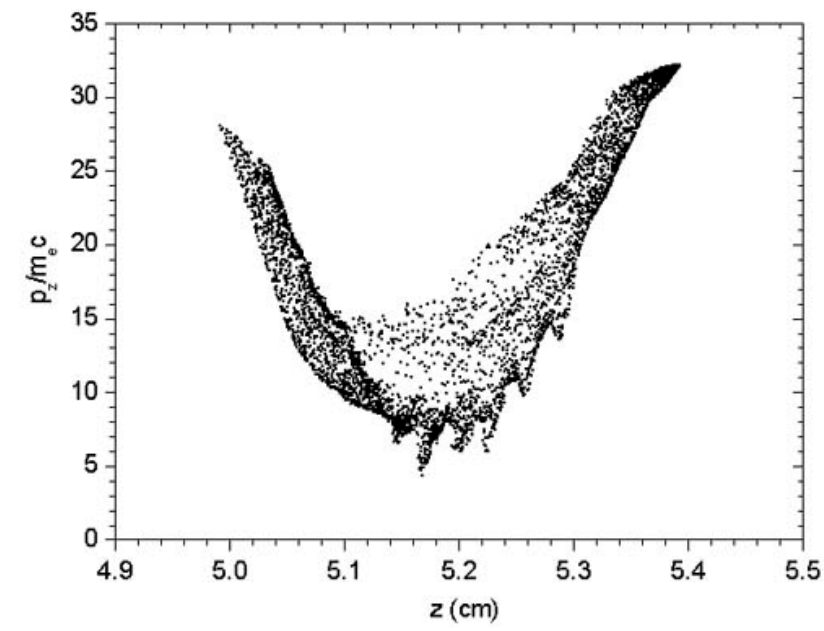

Figure 2: Plot of phase space $\left(p_{z}, z\right)$ for the driver beam. Note that the high frequency instability develops in the low energy range. 
It is well-known that wavelength of the betatron radiation is given by $\lambda_{r}=\sqrt{2} \pi c / \gamma^{3 / 2} \omega_{p}$ and this approximately agrees with the observed oscillation frequency in Fig. 2 . Hence, it is conjectured that the betatron radiation interacts with the beam electrons and eventually this process yields a longitudinal momentum oscillations. The oscillations have a saw-tooth shape as lower energy particles move at a smaller velocity. It should be noted that the instability has a higher growth rate in the low energy range

As the driver beam continues to propagate through the plasma, the beam loses its energy continuously and the instability also grows. Eventually energy of some electrons in the central part of the beam reach almost zero, so they slip to the acceleration phase of the wakefield and they are slightly accelerated. This is shown in Fig. 3. If this phenomenon occurs, the slipped particles mix with the trapped plasma electron beam, which leads to a detrimental effect to the beam quality of the originally trapped plasma electron beam. Hence, the trapped plasma electron beam must be ejected from the background plasma before this kind of mixing occurs. If the beam propagates further, more electrons flow to the acceleration phase of the first period and eventually they slip to the second period again. As the beam loses its energy, a space-charge effect becomes more important and the beam radius increases. This is clearly shown in Fig. 3(b), i.e., the low-energy beam part expands transversely.
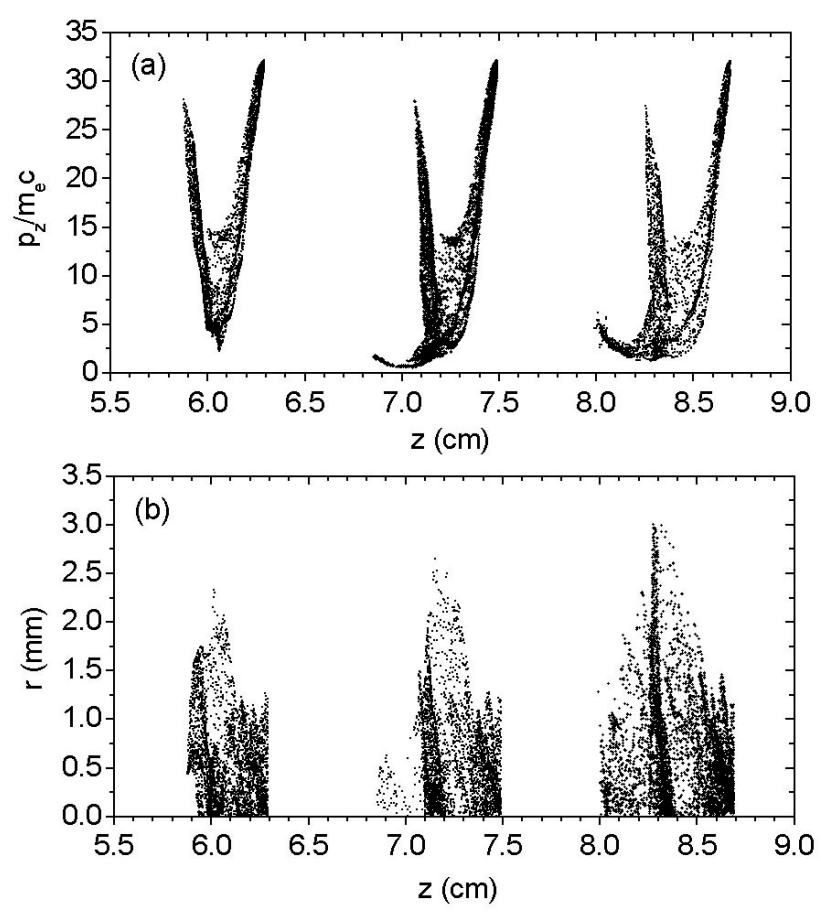

Figure 3: Disruption process of the driver beam. (a) Plot of phase space $\left(p_{z}, z\right)$ for the driver beam. (b) Plot of phase space $(r, z)$ for the driver beam.
The figure also indicates that the driver beam is separated into three bunches longitudinally. If the beam propagates further, the beam is split to more bunches and the beam is disrupted completely.

\section{SUMMARY}

Unlike a long beam, a short beam $\left(L_{b}<\lambda_{p}\right)$ in a plasma does not have low frequency instabilities, but it develops a high frequency instability of which the frequency is much higher than the plasma frequency $\omega_{p}$. It seems that the high frequency instability comes from interactions of the beam and the betatron radiation. The instability develops significantly as the beam loses its energy in the plasma and its growth rate is larger in the low energy range. Hence, some low energy electrons due to the instability begins to flow to the acceleration phase of the wakefield when the driver beam loses enough energy. This process leads to disruption of the driver beam and quality deterioration of the originally trapped plasma electron beam occurs. Hence, the driver beam can not be used for wakefield acceleration any more if this happens and the trapped plasma electron beam must be ejected from the background plasma before this process occurs.

\section{ACKNOWLEDGEMENTS}

One of the authors (H. S.) would like to express deep thanks to Prof. T. Katouleas at USC for the MAGIC code. The work was supported by the U.S. Department of Energy.

\section{REFERENCES}

[1] H. Suk et al., Phys. Rev. Lett. 86, 1011 (2001).

[2] T. Tajima and J. M. Dawson, Phys. Rev. Lett. 43, 267 (1979)

[3] C. Clayton et al., Phys. Rev. Lett. 70, 37 (1993).

[4] J. Rosenzweig et al., Phys. Rev. Lett. 61, 98 (1998).

[5] K. Nakajima et al., Phys. Rev. Lett. 74, 37 (1995).

[6] Bulanov et al., Phys. Rev. E 58, R5257 (1998).

[7] Suk et al., Proc. of the $9^{\text {th }}$ Workshop on Advanced Accelerator Concepts (to appear).

[8] B. Goplen et al., Comput. Phys. Commun. 87, 54 (1995).

[9] E. Esarey et al., IEEE Trans. on Plasma Sci. 24, 252 (1996). 